RAIRO Operations Research

RAIRO Oper. Res. 41 (2007) 213-229

DOI: $10.1051 /$ ro:2007017

\title{
OPTIMIZATION OF POWER TRANSMISSION SYSTEMS USING A MULTI-LEVEL DECOMPOSITION APPROACH
}

\author{
Alexandre Dolgui $^{1}$, Nikolai Guschinsky ${ }^{2}$ \\ AND GENRIKH LEVIN ${ }^{2}$
}

\begin{abstract}
We discuss the use of operations research methods for computer-aided design of mechanical transmission systems. We consider how to choose simultaneously transmission ratios and basic design parameters of transmission elements (diameters, widths, modules and tooth number for gears, diameters of shafts). The objectives, by the order of importance, are: to minimize the deviation of the obtained speeds from desired; to maximize the transmission life; to minimize the total mass. To solve this problem, we propose a multi-level decomposition scheme in combination with methods of quadratic and dynamic programming. Some industrial cases are solved. For these cases, the developed software tool improves the design decisions by decreasing total metal consumption of the transmission as much as $7-10 \%$ and considerable simplifies the work of the designer.
\end{abstract}

Keywords. Power transmission design, optimization, multi-level decomposition, graph approach, quadratic programming, dynamic programming.

Mathematics Subject Classification. 90B10, 90C35, 90C90.

Received September 15, 2005. Accepted April 26, 2006.

${ }^{1}$ Division for Industrial Engineering and Computer Sciences, École Nationale Supérieure des Mines de Saint Etienne, 158, cours Fauriel, 42023 Saint Etienne Cedex, France;

dolgui@emse.fr

${ }^{2}$ United Institute of Informatics Problems, National Academy of Sciences of Belarus, Surganov Str. 6, 220012 Minsk, Belarus

(c) EDP Sciences, ROADEF, SMAI 2007 


\section{IntroduCtion}

In this paper, we present a new and interesting application of the Operational Research. This application concerns computer-aided design of complex mechanical power transmission systems. We show that the choice of transmission ratios and basic design parameters of transmission elements can be formulated as an optimization problem and we develop dedicated optimization methods based on a multi-level decomposition procedure $[14,16]$ and advanced operational research techniques.

Transmission systems are crucial components of many machines and mechanisms. Ken Hurst [10] highlights that whether you're designing power plants, cars, or washing machines, the power transmission system is an integral component responsible for product success or failure.

The components that compose a power transmission system include that which transfer power directly (coupling and shaft), speed/torque multiplication components (gears, belt drives, etc.), and related mechanisms (clutches, brakes, etc.) [4].

Transmission design is a very complex problem. In literature, the main publications concern the design of specific elements such as spring, gears, and shafts, see for example $[13,17,19,23]$. Some methods were suggested for structural and parametric synthesis for some kinds of transmission $[9,13]$.

For the design of complex power transmission systems, the functional decomposition approach is often used [5]. Here, a complex transmission system is decomposed to a set of basic elements, and each basic element is then separately optimized. To take into account external conditions for components, complementary constraints are added [1].

Expert systems are widely used in order to consider engineering experience and to integrate the partial optimization models in the design process [2, 20-22].

Another method deals with hierarchical decomposition $[11,18]$. The optimization model is decomposed and solved as a set of smaller, coordinated sub-problems. Such a process is often followed intuitively, during the development of this model, by adding together selected objectives of each sub-system to obtain an overall system objective.

In this paper, we focus on the preliminary design stage of transmission systems with a complex structure. These systems consist of a lot of shafts and gears (between 20 and 60), and the number of kinematic chains (speeds) can be greater than 60 . Decisions at this stage are pivotal since the main design parameters of transmission system are predetermined. These decisions can be completed during next stages of design.

The remainder of the paper is organized as follows. Section 2 gives a mathematical programming formulation of the problem considered. In Section 3, the multi-level decomposition approach and the proposed optimization methods are presented. Section 4 shows the results of optimization for some real life transmission systems. Section 5 gives some conclusion remarks. 


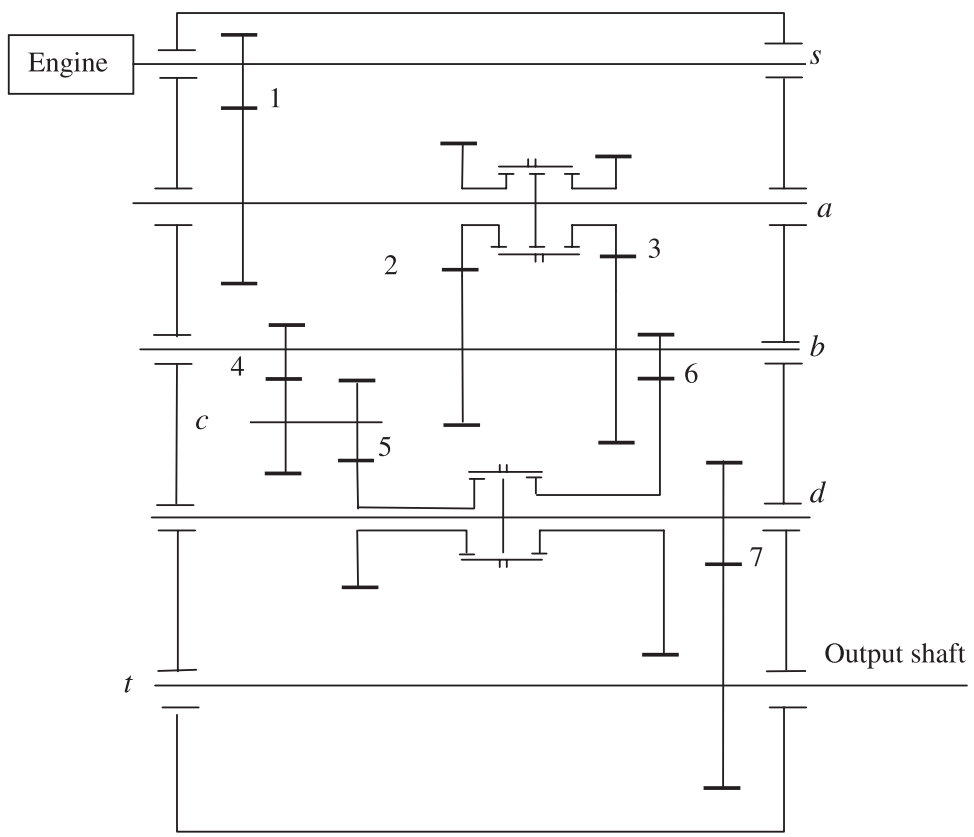

Figure 1. An example of power transmission system.

\section{Optimization PROBLEM}

\subsection{Problem statement}

Power transmission systems include chains of various gears which transmit power and motion from the engine to the output shaft of the transmission. An example of a power transmission system is given in Figure 1.

The system includes six shafts $\{\mathrm{s}, a-d, t\}$ and seven gears $\{1-7\}$. Input shaft $s$ is connected to the engine. The number of different speeds of the output shaft $t$ is equal to four. Each speed corresponds to a kinematic chain (a sequence of gears and shafts from the engine to the output shaft). To select a speed of the output shaft $t$ we need to choose between gear 2 and gear 3 for shaft $a$ as well as between gear 5 and gear 6 , for shaft $d$. The speed depends on transmission ratios of selected gears (kinematic chain). So, changing transmission ratio of a gear modifies the speeds for all kinematic chains involved.

Possible transmission ratios for a gear are defined by the range of their available values and also by feasible speeds of the corresponding shafts. When designing a power transmission system, the nominal speeds of the output shaft as well as the nominal speed of the engine are given. It is necessary to choose a transmission ratio for each gear in such a way that all the desired nominal speeds of output shaft are provided as closely as possible. Actually, these speeds cannot be obtained 
precisely due to intersections of the kinematic chains (when we change a gear ratio for a chain, the speeds of other chains which use the same gear are also modified).

Other important considerations for transmission systems are their mass and lifetime. The mass of transmission elements (gear, shaft) is defined by its design parameters (diameters, widths, modules and tooth number for gear, diameters of shafts). As for the transmission lifetime, it depends on the design parameters and load conditions.

The objective is to choose design parameters providing:

(i) minimal deviation of the output nominal speeds from the desired values, then:

(ii) the maximum lifetime,

and finally:

(iii) the minimum mass of the transmission system.

We consider the problem of determining the following design parameters:

- gear transmission ratios;

- basic design parameters of transmission elements (for gears: types, diameters, widths; for shafts: diameters, etc.).

The input data is:

- the desired nominal speeds of the output shaft;

- load conditions and total working times of the output shaft for each kinematic chain;

- ranges of possible values for transmission ratios of gears;

- ranges of possible values for absolute speeds of shafts;

- total transmission life (taking into account contact and bending fatigue of gears and static rolling strength of shafts);

- characteristics of materials used.

In this paper, we suppose that the structural scheme of the transmission (or some variants of this scheme) has been selected at previous design stages and that the load conditions of actuator are known. We assume that the total transmission life is principally determined by the lifetime of "bottleneck" element.

\subsection{Mathematical Model}

We use a finite acyclic directed multigraph $G=(V, E)$ for representing the structural scheme of the transmission. This digraph has one initial node $s$ and one terminal node $t$. These nodes correspond to the input shaft of the transmission (for example, the engine shaft) and the output shaft, respectively. The nodes $v \in V^{\prime}=V \backslash\{s, t\}$ represent intermediate shafts of the transmission, arcs from $E$ correspond to the gears of the transmission. Each kinematic chain from the input shaft $s$ to the shaft $v \in V \backslash\{s\}$ defines in one-to-one manner a path in $G$ from node $s$ to node $v$.

For the example of transmission given in Figure 1, the digraph $G$ is shown in Figure 2. 


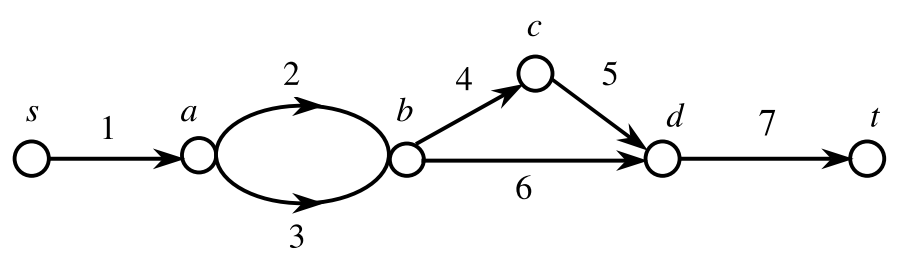

FIGURE 2. Digraph of the structural scheme of transmission.

Let us denote:

- a given nominal speed of the engine (input shaft $s$ ) by $n_{0}$;

- an unknown transmission ratio of the gear $e \in E$ and its range by $x(e)$, and $[\underline{x}(e), \bar{x}(e)] \in \mathbf{R}$;

- a given range of absolute speeds of the shaft $v \in V$ by $[\underline{n}(v), \bar{n}(v)]$;

- a collection of unknown design parameters of the gear $e \in E$ and a set of its feasible values by $u(e)$ and $U(e)$;

- a collection of unknown design parameters of the shaft $v \in V$ and a set of its feasible values by $w(v)$ and $W(v)$;

- input and output shafts of the gear $e \in E$ by $v_{1}(e)$ and $v_{2}(e)$;

- the set of paths in $G$ from node $s$ to node $v$ by $P(v)=\left\{p_{k}(v) \mid k=1,2\right.$, $\ldots, r(v)\}$

- the desired speed of the output shaft for the kinematic chain $p_{k}(t)$ by $C_{k}$, $k=1,2, \ldots, r(t)$.

The speed of shaft $v \in V$ for the kinematic chain $p_{k}(v) \in P(v)$ and fixed $x(e)$, $e \in p_{k}(v)$, is defined as follows:

$$
n_{k}(v, x)=\frac{n_{0}}{\prod_{e \in p_{k}(v)} x(e)} .
$$

Here and later in the paper:

$$
\begin{aligned}
x & =(x(e) \mid e \in E) ; \\
X & =\{x \mid x(e) \in[\underline{x}(e), \bar{x}(e)]\} ; \\
N(v, x) & =\left(n_{1}(v, x), \ldots, n_{r(v)}(v, x)\right) ; \\
C & =\left(C_{1}, \ldots, C_{r(t)}\right) ; \\
u & =(u(e) \mid e \in E) ; \\
w & =(w(v) \mid v \in V) .
\end{aligned}
$$

For the given engine power, the load conditions of the output shaft of the transmission system and each value from $C$, we assume that load conditions of the shaft $v \in V$ and of the gear $e \in E$ can be defined by collections $N(v, x)$ and $\left(x(e), N\left(v_{1}(e), x\right)\right)$, respectively. Based on well-known methods for calculating 
transmission elements (see for example $[13,17,19]$ ), we can calculate the values of the following functions:

(i) functions $T_{v}(N(v, x), w(v))$ and $T_{e}\left(x(e), N\left(v_{1}(e), u(e)\right)\right)$ which determine the longevity of the shaft $v \in V$ and the longevity of the gear $e \in E$ for fixed values of unknown parameters $x, w$ and $u$;

(ii) functions $M_{v}(w(v))$ and $M_{e}(u(e))$ which determine the mass of the shaft $v \in V$ and the mass of the gear $e \in E$ for fixed values of unknown parameters $w$ and $u$.

For the method developed in this paper, we consider the functions $(i),(i i)$ as a sort of black box. To simplify the presentation, in the remaining text (when it is possible) we will use for these functions the notation $T_{v}, T_{e}, M_{v}$ and $M_{e}$, i.e. without parameters.

A transmission ratio is the ratio of the tooth numbers of 2 corresponding toothed wheels. In real life problems, teeth numbers are usually varied from 12 to 100 . So, for any desired transmission ratio, we can find the corresponding number of teeth for each wheel with a sufficient, for this design stage, precision. Thus, we can relax the constraint on the discreetness of possible gear transmission ratio values caused by the integrality of teeth numbers. Moreover, we relax the interdependence between the longevities of the transmission elements; this will be taken into account in following design stages.

From practical point of view, and taking into account the design specificity, the main criterion is the deviation of the output nominal speeds from the desired values. The second criterion in the order of importance is the lifetime, and the last criterion is the mass of the transmission system.

Therefore, the considered design problem is reduced to the following multicriteria optimization problem with lexicographical order of criteria:

$$
\begin{aligned}
& \operatorname{Min}_{1}(x)=\sum_{k=1}^{r(t)} \alpha_{k}\left(\ln \left(C_{k}\right)-\ln \left(n_{k}(t, x)\right)\right)^{2}, \\
& \operatorname{Max} g_{2}(x, u, w)=\min \left(\min \left\{T_{v} \mid v \in V\right\}, \min \left\{T_{e} \mid e \in E\right\}\right), \\
& \operatorname{Min} g_{3}(u, w)=\sum_{e \in E} M_{e}+\sum_{v \in V} M_{v},
\end{aligned}
$$

subject to:

$$
\begin{aligned}
& x \in X, \\
& n_{k}(v, x) \in[\underline{n}(v), \bar{n}(v)], v \in V, k=1,2, \ldots, r(v), \\
& w(v) \in W(v), v \in V, \\
& u(e) \in U(e), e \in E .
\end{aligned}
$$

Here the coefficients $\alpha_{k}(t), k=1,2, \ldots, r(t)$ characterize the relative importance of desired values $C_{k}$ of speeds. The first criterion (1) is to minimize the deviation of the obtained speeds from the desired speeds. The second (2) is to maximize the 
transmission life, and the third (3) is to minimize the total mass of the transmission.

\section{Optimization APPROACH}

\subsection{Decomposition scheme}

The problem (1-7) is a optimization problem where the functions $T_{v}, T_{e}, M_{v}$ and $M_{e}$ are defined algorithmically. To solve this problem, we propose the following multilevel decomposition:

1. At the first stage, we minimize the function $g_{1}(x)$ over a set of all feasible $x$ satisfying kinematic constraints (4), (5). As a result, a vector $C^{*}=\left(C_{k}^{*}=\right.$ $\left.n_{k}\left(t, x^{*}\right) \mid k=1,2, \ldots, r(t)\right)$ (set of output shaft speeds) which is the nearest vector to the vector $C$ is determined (Problem A). This Problem A can be solved several times with different coefficients $\alpha_{k}(t)$. These coefficients are chosen each time by the designer after informal analysis of the obtained solution. Finally, the accepted solution (vector $C^{*}$ ) is used at the second stage as the set of nominal speeds of the output shaft to be implemented by the transmission system.

2. At the second stage, we use an epsilon-constraint approach. The designer specifies a set $\Delta=\left\{\tau_{1}, \tau_{2}, \ldots\right\}$ of acceptable values of the total transmission life and for each value $\tau \in \Delta$ optimal (according to the total mass of the transmission) values of unknown parameters are defined by minimizing the function $g_{3}(u, w)$ subject to $g_{2}(x, u, w) \geqslant \tau$. Simultaneously we assure that the set of the obtained speeds is exactly equal to the set $C^{*}$ obtained during the first stage (Problem B). The most suitable combination of $\tau$ and $g_{3}^{*}(\tau)$ is selected, where $g_{3}^{*}(\tau)$ is the optimal value of function $g_{3}(u, w)$ for fixed $\tau$.

\subsection{Problem A}

For solving problem A, we transform it into logarithmic coordinates by change of variables $z(e)=\ln x(e)$ :

$$
\begin{aligned}
& \operatorname{Min}_{1}(x)=\hat{g}_{1}(z)=\sum_{k=1}^{r(t)} \alpha_{k}\left[\ln \left(C_{k}\right)-\ln n_{0}+\sum_{e \in p_{k}(t)} z(e)\right]^{2}, \\
& z(e) \in[\ln \underline{x}(e), \ln \bar{x}(e)], e \in E, \\
& \sum_{e \in p_{k}(v)} z(e) \geqslant \ln n_{0}-\ln \bar{n}(v), v \in V, k=1, \ldots, r(v), \\
& \sum_{e \in p_{k}(v)} z(e) \leqslant \ln n_{0}-\ln \underline{n}(v), v \in V, k=1, \ldots, r(v) .
\end{aligned}
$$


It is easy to see that the constraints (9)-(11) are not compatible if

$$
\bar{\mu}(v)=\min \left\{\sum_{e \in p_{k}(v)} \ln \bar{x}(e) \mid k=1, \ldots, r(v)\right\}<\ln n_{0}-\ln \bar{n}(v)
$$

or

$$
\underline{\mu}(v)=\max \left\{\sum_{e \in p_{k}(v)} \ln \underline{x}(e) \mid k=1, \ldots, r(v)\right\}>\ln n_{0}-\ln \underline{n}(v) .
$$

The values $\underline{\mu}(v)$ and $\bar{\mu}(v)$ can be found by using shortest (or critical) path algorithm for acyclic digraph $G$ with arc lengths $\ln \bar{x}(e)$ and $\ln \underline{x}(e)$, respectively.

The problem (8)-(11) is a quadratic programming problem with linear constraints. The number of each of constraints (10)-(11) in real life problems can be sufficiently large and in general is equal to the number of all paths in digraph $G$ from the vertex $s$ to all other vertices. However, as a rule, the number of active constraints is no more than $\left|V^{\prime}\right|$. For example, for the digraph of a tractor transmission system with 17 shafts and 27 gears the number of variables is equal to 27 and the number of constraints (10)-(11) can be more than 800 .

For solving the problem A, we use an overall scheme of constraint relaxation [15]. Initially, this problem is solved without constraints (10)-(11), and then we add step by step the constraints that are not valid for the solution obtained for the previous set of constraints. For fixed set of constraints, a current sub-problem is solved by well-known methods of quadratic programming. At each step for each vertex $v$, we add only those invalid constraints (10)-(11), which have the greatest discrepancies. These paths (which correspond to such constraints) can be also found by a shortest path algorithm.

\subsection{Problem B}

The problem B is to minimize the function $g_{3}(u, w)$ for fixed $\tau \in \Delta$ and $C_{k}^{*}$, $k=1, \ldots, r(t)$, under constraints $(4)-(7)$ and the additional constraints:

$$
\begin{aligned}
& g_{2}(x, u, w) \geqslant \tau, \\
& N(t, x)=C^{*} .
\end{aligned}
$$

For solving the problem B, we construct on the base of the mentioned above functions $T_{v}, T_{e}, M_{v}$ and $M_{e}$ the procedures for determining

$$
M_{v}^{*}=M_{v}^{*}(N(v, x))=\min \left\{M_{v} \mid T_{v} \geqslant \tau, w(v) \in W(v)\right\}
$$

and

$$
M_{e}^{*}=M_{e}^{*}\left(x(e), N\left(v_{1}(e), x\right)\right)=\min \left\{M_{e} \mid T_{e} \geqslant \tau, u(e) \in U(e)\right\} .
$$


Then the problem B can be stated as follows:

$$
\begin{aligned}
& \operatorname{Min} g(x)=\sum_{e \in E} M_{e}^{*}+\sum_{v \in V} M_{v}^{*} \\
& x \in X, \\
& n_{k}(v, x) \in[\underline{n}(v), \bar{n}(v)], v \in V, k=1,2, \ldots, r(v), \\
& n_{k}(t, x)=C_{k}^{*}, k=1,2, \ldots, r(t) .
\end{aligned}
$$

For solving the problem B, we propose a method based on a system of invariants. Since the speeds of the output shaft are fixed, then the speeds of the same intermediate shaft are interconnected. The following theorem establishes this property.

Theorem. For any $x \in X, v \in V$ and $e \in E$ values $n_{k}(v, x)=n_{1}(v, x) \bullet \lambda_{k}(v)$, $k=1, \ldots, r(v), x(e)=n_{1}\left(v_{1}(e), x\right) / n_{1}\left(v_{2}(e), x\right) / \gamma(e)$, and $n_{k}(v, x \times \gamma)=n_{1}(v, x)$, where $\lambda_{k}(v)$ and $\gamma(e)$ are constants.

Here $x \times \gamma$ is component-wise multiplication of vectors $x$ and $\gamma$. The proof of this theorem can be found in [8].

Values $\lambda_{k}(v), \gamma(e)$ for all $v \in V$ and $e \in E$ can be determined before solving the problem B. Without loss of generality, we can assume that paths in the sets $P(v)$ for all $v \in V$ are enumerated in a such a way that for any $v \in V$ and $k^{\prime}, k^{\prime \prime} \in\{1, \ldots, r(v)\}$ sub-paths from $v$ to $t$ in paths $p_{m\left(v, k^{\prime}\right)}(t)$ and $p_{m\left(v, k^{\prime \prime}\right)}(t)$ coincide. Here $m(v, k)$ is the minimal number from $\{1, \ldots, r(t)\}$ such that path $p_{k}(v)$ is a sub-path of $p_{m(v, k)}(t)$. Then, $\lambda_{k}(v)=C_{m(v, k)}^{*} / C_{m(v .1)}^{*}$ and $\gamma(e)=\lambda_{l(e)}\left(v_{2}(e)\right)$, where $l(e)$ is the index of the path $\left(p_{1}\left(v_{1}(e)\right), e\right)$ in the set $P\left(v_{2}(e)\right)$.

Let us introduce new variables $y(v)=n_{1}(v, x)$ for all $v \in V$. Then, the problem B can be reformulated as follows:

$$
\begin{aligned}
& \operatorname{Min} \sum_{e \in E} f_{e}^{*}(y)+\sum_{v \in V} h_{v}^{*}(y) \\
& y\left(v_{1}(e)\right) / y\left(v_{2}(e)\right) / \gamma(e) \in[\underline{x}(e), \bar{x}(e)], \quad e \in E, \\
& y(v) \in[\underline{y}(v), \bar{y}(v)], \quad v \in V, \\
& y(s)=n_{0}, \\
& y(t)=C_{1}^{*}
\end{aligned}
$$

where

$$
\begin{aligned}
& f_{e}^{*}(y)= M_{e}^{*}\left(y\left(v_{1}(e)\right) / y\left(v_{2}(e)\right) / \gamma(e), y\left(v_{1}(e)\right), y\left(v_{1}(e)\right) \lambda_{2}\left(v_{1}(e)\right), \ldots,\right. \\
&\left.y\left(v_{1}(e)\right) \lambda_{r^{\prime}}\left(v_{1}(e)\right)\right), \\
& r^{\prime}= r\left(v_{1}(e)\right) \\
& h_{v}^{*}(y)= M_{v}^{*}\left(y(v), y(v) \lambda_{2}(v), \ldots, y(v) \lambda_{r(v)}(v)\right), \\
& \underline{y}(v)= \max \left[\underline{n}(v), e^{\underline{\mu}(v)}\right] / \min \left\{\lambda_{k}(v) \mid k=1, \ldots, r(v)\right\} \\
& \bar{y}(v)=\min \left[\bar{n}(v), e^{\bar{\mu}(v)}\right] / \max \left\{\lambda_{k}(v) \mid k=1, \ldots, r(v)\right\} .
\end{aligned}
$$


Let us denote $V_{c}=\left\{v_{0}=s, v_{1}, v_{2}, \ldots, v_{m}, v_{m+1}=t\right\}$, where nodes $v_{i}, i=1,2$, $\ldots, m$, are cutpoints of the digraph $G$. It is assumed that nodes $v_{i}, i=1,2, \ldots, m$, are ordered in the increasing order of node ranks in the digraph $G$. Recall that a node $v$ is a cutpoint of the $\operatorname{digraph} G$ if and only if there exist nodes $v^{\prime}$ and $v^{\prime \prime}$ such that the node $v$ lies on each path from $v^{\prime}$ to $v^{\prime \prime}$. It should be noted that the nodes $s$ and $t$ can be chosen as $v^{\prime}$ and $v^{\prime \prime}$ since each node in the $\operatorname{digraph} G$ is connected with $s$ and $t$ by a path.

If a digraph $G$ has at least one cutpoint, the graph $G$ is called series decomposable. In such a case, let $G\left(v_{i}, v_{i+1}\right)=\left(V\left(v_{i}, v_{i+1}\right), E\left(v_{i}, v_{i+1}\right)\right), i=0, \ldots, m$, be a graph generated by all the paths from $v_{i}$ to $v_{i+1}$. We can construct a digraph $G\left(V_{c}\right)=\left(V_{c}, E\left(V_{c}\right)\right)$ by replacing each subgraph $G\left(v_{i}, v_{i+1}\right), i=0, \ldots, m$, with an $\operatorname{arc} \varepsilon_{i}=\left(v_{i}, v_{i+1}\right)$.

We assign to the $\operatorname{arc} \varepsilon_{i}$ the range

$$
\begin{aligned}
{\left[\underline{x}\left(\varepsilon_{i}\right), \bar{x}\left(\varepsilon_{i}\right)\right] } & =\left[\max \left\{\prod_{e \in p\left(v_{i}, v_{i+1}\right)} \underline{x}(e) \gamma(e) \mid p\left(v_{i}, v_{i+1}\right) \in P\left(v_{i}, v_{i+1}\right)\right\},\right. \\
& \left.\min \left\{\prod_{e \in p\left(v_{i}, v_{i+1}\right)} \bar{x}(e) \gamma(e) \mid p\left(v_{i}, v_{i+1}\right) \in P\left(v_{i}, v_{i+1}\right)\right\}\right]
\end{aligned}
$$

and $\gamma\left(\varepsilon_{i}\right)=1$, where $P\left(v_{i}, v_{i+1}\right)$ is the set of all paths in $G$ from $v_{i}$ to $v_{i+1}$. As a result, the graph $G\left(V_{c}\right)$ is a path and the problem (12)-(16) can be transformed into the following:

$$
\begin{aligned}
& \operatorname{Min} \sum_{i=0}^{m} F_{i}\left(y\left(v_{i}\right), y\left(v_{i+1}\right)\right)+\sum_{i=0}^{m+1} h_{v}^{*}(y) \\
& y\left(v_{i}\right) / y\left(v_{i+1}\right) / \gamma\left(\varepsilon_{i}\right) \in\left[\underline{x}\left(\varepsilon_{i}\right), \bar{x}\left(\varepsilon_{i}\right)\right], \quad i=0, \ldots, m, \\
& y(v) \in[\underline{y}(v), \bar{y}(v)], \quad v \in V_{c}, \\
& y\left(v_{0}\right)=n_{0}, \\
& y\left(v_{m+1}\right)=C_{1}^{*},
\end{aligned}
$$

where

$$
\begin{array}{r}
F_{i}\left(y^{\prime}, y^{\prime \prime}\right)=\min \left\{\sum_{e \in E\left(v_{i}, v_{i+1}\right)} f_{e}^{*}(y)\right. \\
+\sum_{v \in V\left(v_{i}, v_{i+1}\right) \backslash\left\{v_{i}, v_{i+1}\right\}} h_{v}^{*}(y) \mid y(v) \in[\underline{y}(v), \bar{y}(v)], v \in V_{c} \backslash\left\{v_{i}, v_{i+1}\right\}, \\
\left.y\left(v_{i}\right)=y^{\prime}, y\left(v_{i+1}\right)=y^{\prime \prime}\right\} .
\end{array}
$$




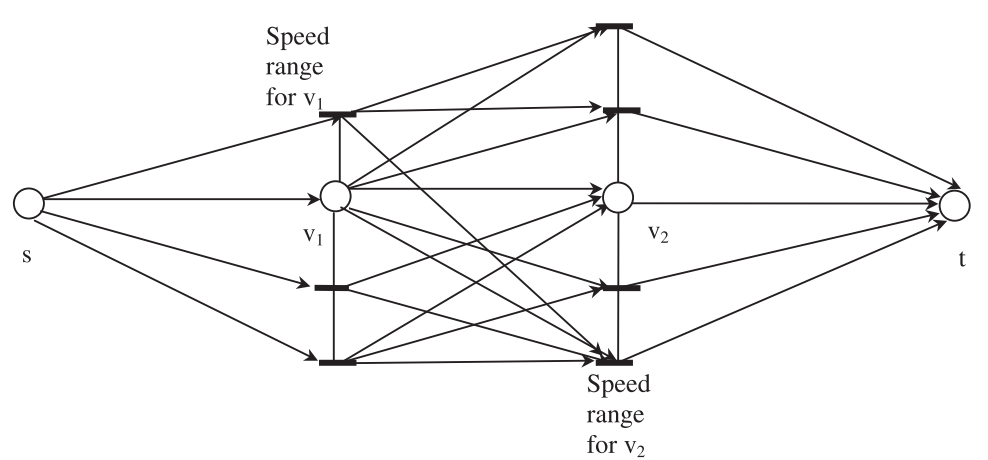

FiguRE 3. Digraph $H$ of possible shaft speeds.

For solving the problem (17)-(21), we transform the digraph $G\left(V_{c}\right)$ into another digraph $H$ of possible shaft speeds, where each vertex of $G\left(V_{c}\right)$ is replaced by a set of vertices of digraph $H$ (see Fig. 3).

Each vertex of digraph $H$ corresponds to the speed of the shaft respectively to the replaced vertex of the digraph $G$. All the vertices of the digraph $H$, obtained from adjacent vertices of the digraph $G$, are connected by an arc. In order to obtain vertices of the digraph $H$, the corresponding speed ranges are discretized. Therefore, the size of the digraph $H$ depends on the discretization factor.

Thus, the problem (17)-(21) is reduced to a shortest path problem in the digraph $H$. We solve this problem using a dynamic programming method.

The problem of calculating $F_{i}\left(y^{\prime}, y^{\prime \prime}\right)$ for fixed $y^{\prime}$ and $y^{\prime \prime}$ is of the same nature as the problem (12)-(16) but its dimension is smaller. Under made transformations, there is no a cutpoint in the digraph $G\left(v_{i}, v_{i+1}\right)$. However, the digraph $G\left(v_{i}, v_{i+1}\right)$ can be parallel decomposable, i.e. the set of all paths $P\left(v_{i}, v_{i+1}\right)$ from $v_{i}$ to $v_{i+1}$ can be divided into minimal (in the sense of inclusion) subsets $P_{j}\left(v_{i}, v_{i+1}\right), j=1,2, \ldots, r\left(v_{i}, v_{i+1}\right)>1$ such that paths from different subsets do not have a common intermediate node. The problem with parallel decomposable digraph is decomposed into $r\left(v_{i}, v_{i+1}\right)$ independent sub-problems with digraphs $G_{j}\left(v_{i}, v_{i+1}\right)=\left(V_{j}\left(v_{i}, v_{i+1}\right), E_{j}\left(v_{i}, v_{i+1}\right)\right)$ (generated by $\left.P_{j}\left(v_{i}, v_{i+1}\right)\right)$ of a smaller dimension. In this case, $F_{i}\left(y^{\prime}, y^{\prime \prime}\right)$ is equal to

$$
\begin{aligned}
& \sum_{j=1}^{r\left(v_{i}, v_{i+1}\right)}\left[\operatorname { m i n } \left\{\sum_{e \in E_{j}\left(v_{i}, v_{i+1}\right)} f_{e}^{*}(y)+\sum_{v \in V_{j}\left(v_{i}, v_{i+1}\right) \backslash\left\{v_{i}, v_{i+1}\right\}} h_{v}^{*}(y) \mid\right.\right. \\
& \left.\left.y(v) \in[\underline{y}(v), \bar{y}(v)], v \in V_{j}\left(v_{i}, v_{i+1}\right) \backslash\left\{v_{i}, v_{i+1}\right\}\right\}, y\left(v_{i}\right)=y^{\prime}, y\left(v_{i+1}\right)=y^{\prime \prime}\right] .
\end{aligned}
$$

Such decomposition cases are illustrated in Figure 4. 


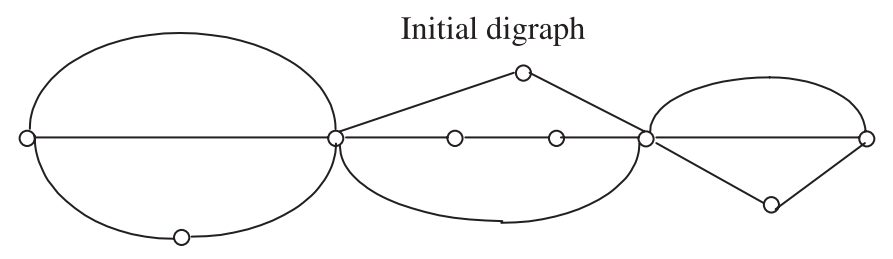

Result of decomposition

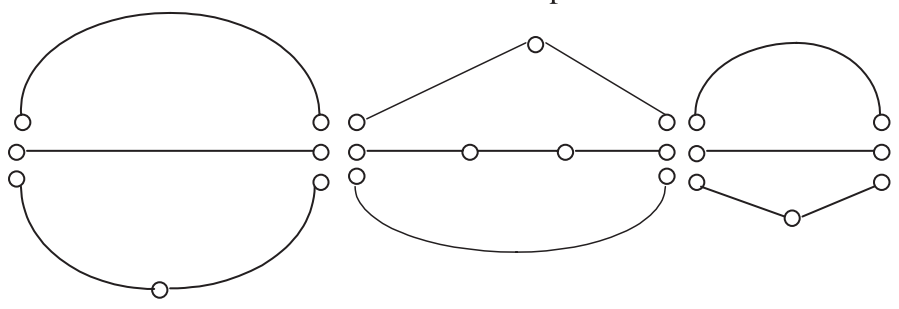

FiguRE 4. Decomposition of digraph $G$.

TABLE 1. Ranges of transmission ratios of gears and nominal speeds of intermediate shafts.

\begin{tabular}{ccccccccccccc}
\hline Gears & 1 & 2 & 3 & 4 & 5 & 6 & 7 & Shafts & $a$ & $b$ & $c$ & $d$ \\
\hline$\underline{x}(e)$ & 2.5 & 1.5 & 2.5 & 2.5 & 2.5 & 2.5 & 3.0 & $\underline{n}(v)$ & 10 & 10 & 10 & 10 \\
$\bar{x}(e)$ & 3.5 & 2.5 & 3.5 & 3.5 & 3.5 & 3.5 & 4.5 & $\bar{n}(v)$ & 3000 & 3000 & 3000 & 3000 \\
\hline
\end{tabular}

\section{INDUSTRIAL EXAMPLES}

First, we give the calculation results for the transmission system considered in Section 2 (see Fig. 1). The corresponding digraph $G$ was presented in Figure 2.

For this example, the following input data is used:

- the power of the engine is $70 \mathrm{~kW}$ and the nominal speed of the input shaft of the transmission is $3000 \mathrm{rpm}$;

- all gears are spurs;

- the length of the shaft $c$ is $150 \mathrm{~mm}$ and the length of others is $450 \mathrm{~mm}$;

- the ranges of transmission ratios of gears and the ranges of nominal speeds of all intermediate shafts are given in Table 1 ;

- composition of kinematic chains, their desired nominal speeds and the loading conditions of the output shaft of the transmission are given in Table 2 .

For strength calculation of gears and shafts the following characteristics of materials are used:

- the hardness of gear teeth is $59 \mathrm{HRC}$;

- the contact fatigue limit is $420 \mathrm{MPA}$;

- the bending fatigue limit is 1130 MPA;

- the yield point of shafts is $580 \mathrm{MPA}$. 
TABLE 2. Nominal speeds and loading conditions of the transmission system.

\begin{tabular}{ccccc}
\hline $\begin{array}{c}\text { Chain } \\
\text { number }\end{array}$ & $\begin{array}{c}\text { Kinematic } \\
\text { chains }\end{array}$ & $\begin{array}{c}\text { Nominal } \\
\text { speeds, rpm }\end{array}$ & $\begin{array}{c}\text { Loads, } \\
\text { Nm }\end{array}$ & $\begin{array}{c}\text { Shares } \\
\text { of time, \% }\end{array}$ \\
\hline 1 & 12457 & 20 & 30000 & 23 \\
2 & 1267 & 50 & 10000 & 22 \\
3 & 13457 & 12 & 45000 & 25 \\
4 & 1367 & 35 & 18000 & 30 \\
\hline
\end{tabular}

TABLE 3. The solutions of problem A for different importance coefficients $\alpha_{k}$.

\begin{tabular}{ccccccccc}
\hline $\begin{array}{c}\text { Chain } \\
\text { number }\end{array}$ & $\begin{array}{c}\text { Kinematic } \\
\text { chains }\end{array}$ & $\begin{array}{c}\text { Nominal } \\
\text { speeds, rpm }\end{array}$ & Solution 1 & Solution 2 & Solution 3 \\
\hline 1 & 12457 & 20 & 1 & 19.24 & 1 & 19.02 & 1 & 18.64 \\
2 & 1267 & 50 & 1 & 51.96 & 1 & 52.54 & 10 & 50.35 \\
3 & 13457 & 12 & 1 & 12.47 & 1 & 12.61 & 1 & 12.87 \\
4 & 1367 & 35 & 1 & 33.67 & 10 & 34.82 & 10 & 34.75 \\
\hline
\end{tabular}

Results of solving the problem A for three different collections of importance coefficients $\alpha_{k}$ are presented in Table 3. By changing coefficients $\alpha_{k}$, we can control the closeness of some components of vector $C^{*}$ to the desired values thanks to others.

The solution of the problem B is illustrated only for the first obtained vector $C^{*}=\{19.24,51.96,12.47,33.67\}$. For this vector $C^{*}$ invariants $\lambda_{k}(v)$ and $\gamma(e)$ are the following: $\lambda_{2}(b)=0.648, \lambda_{2}(c)=0.648, \lambda_{2}(d)=2.701, \lambda_{3}(d)=0.648, \lambda_{4}(d)=$ $1.75, \gamma(3)=0.648, \gamma(6)=2.701$. Other invariants are equal to 1 . Results of solving the problem $B$ for three values of the total transmission life $\{3000,5000,7500\}$ are given in Table 4. In this table $\mathrm{d}_{1}(e)$ and $\mathrm{d}_{2}(e)$ are diameters of drive and driven wheels of gear $e, \psi(e)$ is the width of gear ring. In each cell of the table three values of design parameters corresponding to different values of the total transmission life are separated by slash $(/)$. The total mass of gears and shafts for $\tau=3000$ is equal to $424 \mathrm{~kg}$, for $\tau=5000$ is $440 \mathrm{~kg}$ and for $\tau=7500$ is $455 \mathrm{~kg}$.

The problem B is usually the most time-consuming in the suggested decomposition approach. In Table 5 we give the time of solving this problem for industrial examples (with different discretization factor) on PC Pentium IV $2.6 \mathrm{GHz}$ and RAM $512 \mathrm{Mb}$. The ranges of possible shaft speeds were discretized using geometric progression with the factor $q>1$.

The experiments show that usually the discretization factor can be taken as 1.01-1.005. As a rule, subsequent its decreasing does not result in essential improvement of the obtained solution. 
TABLE 4. The design parameters of gears and shafts for $\Delta=\{3000,5000,7500\}$.

\begin{tabular}{ccccccc}
\hline & \multicolumn{4}{c}{ Gear parameters } & \multicolumn{2}{c}{ Shaft } \\
Gears & $x(e)$ & $d_{1}(e)$ & $d_{2}(e)$ & $\psi(e)$ & Shafts & diameter \\
\hline 1 & $3.5 / 3.5 / 3.5$ & $68.1 / 68.1 / 68.1$ & $238 / 238 / 238$ & $27.2 / 27.2 / 27.2$ & $s$ & 20 \\
2 & $2.35 / 2.23 / 2.24$ & $90.1 / 96.5 / 100$ & $212 / 215 / 225$ & $36.0 / 38.6 / 40.2$ & $a$ & 30 \\
3 & $3.48 / 3.29 / 3.30$ & $90.8 / 97.1 / 97.3$ & $316 / 319 / 322$ & $36.3 / 38.8 / 38.9$ & $b$ & 43 \\
4 & $2.89 / 2.99 / 2.97$ & $118 / 116 / 121$ & $343 / 349 / 361$ & $47.4 / 46.6 / 48.5$ & $c$ & 59 \\
5 & $2.60 / 2.65 / 2.65$ & $166 / 165 / 165$ & $433 / 439 / 439$ & $66.6 / 66.2 / 66.2$ & $d$ & 82 \\
6 & $2.71 / 2.86 / 2.85$ & $125 / 122 / 125$ & $339 / 352 / 359$ & $50.0 / 49.1 / 50.3$ & $t$ & 117 \\
7 & $3 / 3 / 3$ & $219 / 223 / 226$ & $658 / 670 / 679$ & $87.7 / 89.4 / 90.6$ & & \\
\hline
\end{tabular}

TABLE 5. Results of solving problem B for industrial examples.

\begin{tabular}{cccccc}
\hline \multicolumn{7}{c}{ Problem size } & $\begin{array}{c}\text { Number of } \\
\text { Number of } \\
\text { gears }\end{array}$ & $\begin{array}{c}\text { Number of } \\
\text { khafts }\end{array}$ & $\begin{array}{c}\text { Discretization } \\
\text { factor }\end{array}$ & $\begin{array}{c}\text { Time } \\
\text { of solution (s) }\end{array}$ & $\begin{array}{c}\text { Quality of solution } \\
\text { (mass, kg) }\end{array}$ \\
\hline 17 & 12 & 24 & $1.01 / 1.005 / 1.001$ & $0.28 / 0.83 / 18.33$ & $50.484 / 50.456 / 50.451$ \\
19 & 14 & 24 & $1.01 / 1.005 / 1.001$ & $0.84 / 3.66 / 206.3$ & $197.34 / 197.19 / 197.18$ \\
14 & 9 & 24 & $1.01 / 1.005 / 1.001$ & $0.44 / 1.59 / 100.2$ & $59.40 / 59.39 / 59.37$ \\
16 & 12 & 16 & $1.01 / 1.005 / 1.001$ & $0.25 / 0.70 / 21.34$ & $315.6 / 315.5 / 315.3$ \\
14 & 9 & 24 & $1.01 / 1.005 / 1.001$ & $0.39 / 1.58 / 99.4$ & $54.536 / 54.537 / 54.531$ \\
19 & 14 & 24 & $1.01 / 1.005 / 1.001$ & $0.84 / 3.67 / 204.3$ & $192.67 / 192.55 / 192.53$ \\
16 & 12 & 16 & $1.01 / 1.005 / 1.001$ & $0.22 / 0.70 / 21.27$ & $304.43 / 304.07 / 303.86$ \\
28 & 18 & 60 & $1.01 / 1.005 / 1.001$ & $0.48 / 2.0 / 125.3$ & $210.89 / 210.87 / 210.84$ \\
27 & 17 & 60 & $1.01 / 1.005 / 1.001$ & $0.44 / 1.94 / 233.6$ & $232.03 / 231.91 / 231.75$ \\
26 & 15 & 72 & $1.01 / 1.005 / 1.001$ & $0.02 / 0.02 / 0.05$ & $255.04 / 255.02 / 255.0$ \\
19 & 14 & 20 & $1.01 / 1.005 / 1.001$ & $0.08 / 0.28 / 11.45$ & $217.78 / 217.36 / 216.89$ \\
18 & 14 & 16 & $1.01 / 1.005 / 1.001$ & $0.09 / 0.28 / 8.01$ & $133.38 / 132.63 / 132.33$ \\
23 & 16 & 36 & $1.01 / 1.005 / 1.0$ & $0.44 .2 .47 / 363.9$ & $178.84 / 178.46 / 178.45$ \\
25 & 17 & 72 & $1.01 / 1.005 / 1.001$ & $0.05 / 0.16 / 2.81$ & $234.29 / 233.97 / 233.39$ \\
\hline
\end{tabular}

\section{Summary AND CONCLUSIONS}

A novel application of operational research techniques is provided. It concerns a vital industrial problem of the optimal design of power transmission systems. A multilevel decomposition approach is used. The models and methods for main sub-problems are developed based on advanced techniques of graph optimization, quadratic and dynamic programming.

The proposed approach permits a good quality approximate solution of the design problem. The developed methods were used for creating a computer aided decision support system of transmission design $[3,6,7]$. This system is oriented to active participation of an experienced designer in decisions. It was tested at Minsk tractor plant for solving real life design problems. The use of this system has confirmed that, during 2-3 sessions on a conventional PC, a designer can analyze and optimize the main design decisions. Such detailed analysis cannot be performed by conventional methods. 
The system improves the design decisions by decreasing total metal consumption of the transmission as much as $7-10 \%$ and considerable simplifies the work of the designer.

\section{REFERENCES}

[1] A. Dadié, Réalisation d'un logiciel de conception automatique appliquée à la détermination des engrenages cylindriques : interfaçage avec un logiciel industriel de C.A.O., Ph.D. thesis, No. 366, INSA, Toulouse (1996).

[2] J.R. Dixon, Knowledge-Based Systems for Design. ASME J. Mech. Design 117 (1995) 1116 .

[3] A. Dolgui, N. Guschinsky and G. Levin, Models and Methods of Multicriteria Optimization for Computer Aided Design of Multi-Unit Mechanical Transmission Systems, in Proc. of the European Control Conference (ECC'99), Karlsruhe, Germany, CD-ROM (1999) 6 p. (and in Summaries Volume, p. 763).

[4] J.S. Freeman and S.A. Velinsky, Design of Vehicle Power Transmission Systems. ASME J. Mech. Design 117 (1995) 113-120.

[5] J. Guillot, Méthodologie de définition des ensembles mécaniques en conception assistée par ordinateur, recherche des solutions optimales, Ph.D. thesis, No. 1343, Université Paul Sabatier, Toulouse (1987).

[6] N. Guschinsky and G. Levin, Optimizing Tree Like Structure Transmission Parameters at Initial Stage of Designing, in Models and Algorithms for CAD/CAM, Minsk, Institute of Engineering Cybernetics (1994) 4-21 (in Russian).

[7] N. Guschinsky and G. Levin, Decision Support System for CAD of Transmissions, in Modeling and Information Technologies in Design. Minsk, Institute of Engineering Cybernetics (1997) 50-57 (in Russian).

[8] N. Guschinsky and G. Levin, Optimization of Quasi-additive Function over Set of Network Arc Parameters. Vesti Academii nauk Belarusi: Physical and Mathematical Science No. 1 (1999) 56-60 (in Russian).

[9] H.I. Hsieh and L.W. Tsai, Kinematic Analysis of Epicyclic-Type Transmission Mechanisms Using the Concept of Fundamental Geared Entities. ASME J. Mech. Design 118 (1996) 295-299.

[10] K. Hurst, Select and Design Optimal Rotary Power Transmission Systems. McGraw-Hill, (1998).

[11] R.S. Krishnamachari and P.Y. Papalambros, Hierarchical Decomposition Synthesis in Optimal Systems Design. ASME J. Mech. Design 119 (1997) 448-457.

[12] V.N. Kudrjavtsev, Machine Elements. Moscow, Mashgis (1980) (in Russian).

[13] V.N. Kudrjavtsev, Y.A. Derjavets and E.G.Glucharev, Design and Calculation of Gear Reducers. Moscow, Machinostroenie (1971) (in Russian).

[14] G.M. Levin and V.S. Tanaev, Decomposition Methods for Optimization of Design Decisions. Minsk, Nauka i technika (1978).

[15] L. Lasdon, Optimization Theory for Large Systems. New York, Macmillan (1970).

[16] G.M. Levin and V.S. Tanaev, Parametric Decomposition of Optimization Problem. Vesti Academii nauk Belarusi: Physical and Mathematical Science No. 4 (1998) 121-131 (in Russian).

[17] F.L. Litvin, Gear Geometry and Applied Theory. Englewood Cliffs, N.J., Prentice Hall, (1994).

[18] N.F. Michelena and P.Y. Papalambros, Optimal Model-Based Decomposition of Powertrain System Design. ASME J. Mech. Design 117 (1995) 499-505.

[19] J.E. Shigley, C.R. Mischke, R.G. Budynas, Mechanical Engineering Design. 7th Ed., McGraw Hill, New York (2003). 
[20] D. Su, Development of Artificial Neural Networks for Conceptual Design of a Power Transmission System, in Proc. 2nd International Conference on Adaptive Computing in Engineering Design and Control. Plymouth, England (1996) 316-318.

[21] D. Su, Intelligent Hybrid System for Integration in Design and Manufacture. J. Mater. Process. Tech. 76 (1998) 23-28.

[22] D. Su, S. Ji, N. Amin and J.B. Hull, Multi-user Internet environment for gear design optimisation. Integr. Manuf. Syst. 14 (2003) 498-507.

[23] D. Su and D. Qin, Integration of numerical analysis, virtual simulation and finite element analysis for optimum design of worm gearing. J. Process. Tech. 138 (2003) 429-435.

\section{Biographical Sketches}

Pr. Alexandre Dolgui is a Full Professor, the Director of the Division for Industrial Engineering and Computer Sciences and the Head of the Department "Scientific Methods for Industrial Management" at the Ecole des Mines de SaintEtienne (France). He obtained Ph.D. degree from United Institute of Informatics Problems of Byelorussian National Academy of Sciences (Belarus) and Dr. Hab. degree from University of Technololy of Compiègne (France). His research focuses on manufacturing line design, production planning, and supply chain optimization. His main results are based on the exact mathematical programming methods and their intelligent coupling with heuristics and meta-heuristics algorithms. He is the author of four books, the editor of one book and 11 conference proceedings, the author of about 85 journal papers and book chapters and over 200 papers in conference proceedings. He is an Associate Editor of the International J. of Systems Science, an Associate Editor of the IEEE Transactions on Industrial Informatics and a Member of the Editorial Board of five other international journals. He has been chairman of several international conferences. He is a Member of the Institute of Industrial Engineers (IIE), the Institute for Operations Research and the Management Sciences (INFORMS) and a Member of the French Operational Research Society (ROADEF).

Dr. Nikolai N. Guschinsky is a Leading Researcher of Operations Research Laboratory at the United Institute of Informatics Problems of Byelorussian National Academy of Sciences (Belarus). Dr. N. Guschinsky received a B.Sc. and M.Sc. in Applied Mathematics from Byelorussian State University and a Ph.D. in Mathematical Cybernetics from Institute of Mathematics of Byelorussian Academy of Sciences. He has been Junior Researcher and Researcher of the Mathematical Cybernetics Laboratory, Senior Researcher of Operation Research Laboratory. He is Byelorussian Operations Research Society Secretary. His research interests include graph optimization and computer-aided design of complex engineering systems.

Pr. Genrikh M. Levin is a Professor and the Head of Operations Research Laboratory at the United Institute of Informatics Problems of Byelorussian National Academy of Sciences (Belarus). Pr. G. Levin received an Engineer degree in Mechanical Engineering from Byelorussian Polytechnic Institute, a Ph.D. in Engineering Cybernetics from United Institute of Informatics Problems of Byelorussian 
National Academy of Sciences (Belarus), and a Doctor of Sciences degree in Computer Science and CAD/CAM from United Institute of Informatics problems of Byelorussian National Academy of Sciences. He is a vice-president of Byelorussian Operations Research Society. His research interests include theory of parametric decomposition, optimization decomposition methods, and discrete optimization, the current applications: $\mathrm{CAD} / \mathrm{CAM} / \mathrm{CAE}$, design of transfer lines, multi-position machines and transmission systems. 